\title{
EFFECTS OF AGE AND ISCHEMIC TIMES ON BIOCHEMICAL EVIDENCE OF MYOCARDIAL INJURY AFTER PEDIATRIC CARDIAC OPERATIONS
}

\author{
D. P. Taggart, MD(Hons), FRCS \\ L. Hadjinikolas, $\mathrm{MD}^{\mathrm{a}}$ \\ J. Hooper, MD, MRCPath \\ J. Albert, FRCS ${ }^{a}$ \\ M. Kemp, MSc, Dip CB, MI Biol ${ }^{\mathrm{b}}$ \\ D. Hue, BSc, $\mathrm{PhD}^{\mathrm{b}}$ \\ M. Yacoub, FRCS ${ }^{\mathrm{a}}$ \\ J. C. Lincoln, FRCS ${ }^{\mathrm{a}}$
}

\begin{abstract}
Introduction: The vulnerability of pediatric myocardium to ischemia is poorly documented in the clinical setting. Methods: Serial measurements of serum concentrations of myoglobin, the MB isoenzyme of creatine kinase, and cardiac troponins $T$ and $I$ and their respective areas under the curve were obtained, with particular reference to age and ischemic time, in $\mathbf{8 0}$ children undergoing cardiac operations. Sixteen (the control group) did not require cardiopulmonary bypass and 64 did. Results: In the control group there were increases $(p<0.01)$ in myoglobin and creatine kinase MB isoenzyme but no increase in cardiac troponin $T$ or $I$; by contrast, the group treated with cardiopulmonary bypass had significant increases in all four markers but with differing temporal patterns. Younger age (especially $<12$ months) was a highly significant explanatory variable only for the release of cardiac troponins $T$ and $I$, and ischemic time was a significant explanatory variable for the release of creatine kinase $\mathrm{MB}$ isoenzyme, cardiac troponins $T$ and I, but not myoglobin. In comparison with previous studies in adults, creatine kinase $\mathrm{MB}$ and cardiac troponin $\mathrm{T}$ concentrations were three times greater in children than in adults. Conclusions: This study supports the specificity of cardiac troponins $T$ and I as markers of myocardial injury after pediatric cardiac operations and defines the importance of age and ischemic time in determining their release. In comparison with previous data in adults, our results raise the possibility that the pediatric heart may be more vulnerable to the effects of ischemia and reperfusion. Cardiac troponins will permit comparison of new myocardial protective strategies or other potentially therapeutic myocardial interventions. (J Thorac Cardiovasc Surg 1997;113:728-35)
\end{abstract}

A dvances in surgical and anesthetic techniques accompanied by refinements in extracorporeal perfusion technology have led to significant improvements in the results of pediatric cardiac surgery during the past decade, prompting efforts at complex operations in increasingly younger children.

The vulnerability of pediatric myocardium to the effects of ischemia and reperfusion in clinical practice is, however, poorly documented. On the basis of

From the Departments of Cardiothoracic Surgery and Biochemistry, ${ }^{\text {b }}$ Royal Brompton Hospital, London, United Kingdom.

Received for publication Jan. 18, 1996; revisions requested June 18, 1996; revisions received Nov. 20, 1996; accepted for publication Nov. 21, 1996.

Address for reprints: D. P. Taggart, MD (Hons), FRCS, Consultant Cardiothoracic Surgeon, John Radcliffe Hospital, Oxford OX3 9DU, United Kingdom.

Copyright (C 1997 by Mosby-Year Book, Inc.

$0022-5223 / 97 \$ 5.00+0 \quad \mathbf{1 2 / 1 / 7 9 4 4 9}$ evidence derived largely from studies on the hearts of healthy young animals, ${ }^{1-5}$ the immature myocardium has traditionally been considered to be more resistant to hypoxia and ischemia than its adult counterpart. There is very little confirmatory data in the clinical setting. However, more than a decade ago Bull, Cooper, and Stark, ${ }^{6}$ in a study of 400 pediatric patients, suggested that half of the postoperative deaths were attributable to inadequate myocardial preservation despite crystalloid cardioplegia. In addition to differences in structure, function, and biochemistry between the healthy immature and mature heart, ${ }^{7}$ the situation is further complicated in patients with congenital heart disease because the myocardium may be volume overloaded or pressure overloaded (or both). Smolenski and colleagues ${ }^{8}$ reported an increased release of lactates, phosphates, and purines in the coronary sinus effluent of children aged 2 to 10 years compared with the levels of these substances in adults during cardiac opera- 
tions. These observations imply that children's hearts are more metabolically vulnerable to the effects of ischemia, cardioplegic arrest, and reperfusion. $\mathrm{We}^{9}$ have recently reported greater elevations of the $\mathrm{MB}$ isoenzyme of creatine kinase (CK-MB) and cardiac troponin $\mathrm{T}$ ( $\mathrm{CTnT}$ ) after pediatric surgery compared with that seen in adults, but we could not evaluate the effects of age and ischemic times.

In the present study, we performed serial measurements of serum concentrations of myoglobin, CK-MB, and the cardiac troponins $\mathrm{T}$ and I (cTnT and $(\mathrm{TnI})$ in 80 consecutive children undergoing operations for congenital heart disease. The study included 16 children undergoing procedures not requiring cardiopulmonary bypass (CPB) (the control group) and 64 children undergoing cardiac operations requiring $\mathrm{CPB}$ (the $\mathrm{CPB}$ group). In particular, we examined the effects of age and ischemic time on the release of these biochemical markers.

\section{Methods}

The study was approved by the Ethical Committee of the Royal Brompton Hospital, and consent obtained from the guardians of the patients.

Patients. The patients comprised 80 children undergoing operations for congenital heart disease, 16 not requiring CPB (the control group) and 64 requiring $\mathrm{CPB}$ (CPB group). The current study includes data from a previous pilot study 9 and the same biochemical measurements in an additional 40 patients.

Surgery. The control patients underwent thoracotomy but no direct cardiac procedure. In the CPB group myocardial protection was obtained with St. Thomas' Hospital cardioplegic solution $(30 \mathrm{ml} / \mathrm{kg})$ at $4^{\circ} \mathrm{C}$ administered under gravity through the aortic root every 30 minutes and additional topical hypothermia with slush ice as necessary. Systemic hypothermia to between $28^{\circ}$ and $32^{\circ} \mathrm{C}$ was used in all patients unless circulatory arrest was required, in which case systemic temperature was reduced to between $15^{\circ}$ and $18^{\circ} \mathrm{C}$.

Blood samples. Serum concentrations of myoglobin, $\mathrm{CK}-\mathrm{MB}, \mathrm{cTnT}$, and $\mathrm{cTnI}$ were measured serially before and $1,6,24$, and 72 hours after the operation. Areas under the curve (AUC), representative of the total release of the biochemical markers, were calculated from the respective curves according to the formula developed by Shell and Sobel. ${ }^{10}$

Biochemical analysis. Biochemical analysis has been described previously. ${ }^{9}$ Myoglobin was measured with a double antibody radioimmunoassay technique (Myoglobin RIA Test Kit, Biogenesis, Bournemouth, United Kingdom); CK-MB was directly measured by microparticle enzyme immunoassay with the ABBOTT IMX CK-MB kit (Abbott Diagnostics Division, Maidenhead, United Kingdom); cTnT and cTnI were both measured by enzyme-linked immunoadsorbent assays (Elisa Troponin
Table I. Explanatory variables for the postoperative release of the biochemical markers in the $C P B$ group $(n=64)$

\begin{tabular}{|c|c|c|c|}
\hline Variable & Median & $Q 25$ & $Q 75$ \\
\hline Age (mo) & 7 & 4 & 48 \\
\hline Ischemic time ( $\mathrm{min}$ ) & 36 & 24 & 56 \\
\hline $\mathrm{CPB}$ time (min) & 59 & 50 & 86 \\
\hline Temperature $\left({ }^{\circ} \mathrm{C}\right)$ & 23 & 15 & 30 \\
\hline $\begin{array}{l}\text { Total circulatory arrest } \\
\qquad(\text { yes }=1, \text { no }=0)\end{array}$ & $48 \%$ & & \\
\hline $\begin{array}{l}\text { Atriotomy } \pm \text { ventriculot- } \\
\text { omy }(\text { yes }=1, \text { no }=0)\end{array}$ & $69 \%$ & & \\
\hline \multicolumn{4}{|l|}{ Operation } \\
\hline $\begin{array}{l}\text { Atrial septal defect } \\
\quad(\text { yes }=1, \text { no }=0)\end{array}$ & $19.7 \%$ & & \\
\hline $\begin{array}{l}\text { Ventricular septal defect } \\
\quad(\text { yes }=1, \text { no }=0)\end{array}$ & $34.4 \%$ & & \\
\hline $\begin{array}{l}\text { Tetralogy of Fallot } \\
\qquad(\text { yes }=1, \text { no }=0)\end{array}$ & $8.2 \%$ & & \\
\hline $\begin{array}{l}\text { Atrial switch operation } \\
\quad(\text { yes }=1, \text { no }=0)\end{array}$ & $18.0 \%$ & & \\
\hline Other $($ yes $=1$, no $=0)$ & $19.7 \%$ & & \\
\hline
\end{tabular}

Q25 and Q75, Lower and upper quartiles.

T kit, Boehringer Mannheim, United Kingdom, and Troponin I Pasteur kit, Sanofi Diagnostics Pasteur Ltd).

Statistics. Data were analyzed with the STATGRAPHICS statistical program (STATGRAPHICS, version 6.0, Manugistics, Inc.). The Mann-Whitney $U$ test was used for the comparison of continuous data between groups and Fisher's exact test for categoric data. Forward stepwise multiple regression analysis was used to identify explanatory variables that influenced the postoperative release of biochemical markers, as expressed by the AUC developed from the formula by Shell and Sobel. ${ }^{10}$

Explanatory variables are summarized in Table I. In the multiple regression model the AUC of biochemical markers, age, and ischemia time underwent a logarithmic transformation as dictated by the analysis of residuals. Results are expressed as median and upper/lower quartiles.

Differences were considered statistically significant at a probability level of $p<0.05$.

\section{Results}

Patients. Clinical data regarding the control and CPB groups are provided in Table II. The median (and upper and lower quartiles) age of the control patients was 3 months ( 1 and 7 months) and that of the CPB group 7 months ( 4 and 48 months) $(p<$ $0.01)$.

Operations. Operations performed in the nonCPB group included six repairs of coarctation, four closures of patent ductus arteriosus, five modified Blalock-Taussig shunts, and one banding of the pulmonary artery. 
Table II. Comparative clinical data between control and $C P B$ groups

\begin{tabular}{lclc}
\hline \multicolumn{1}{c}{ Variable } & $\begin{array}{c}\text { Control group } \\
(n=16)\end{array}$ & $\begin{array}{l}\text { CPB group } \\
(n=64)\end{array}$ & $p$ Value \\
\hline Age (mo) & $3(1-7)$ & $7(4-48)$ & 0.0117 \\
Postoperative ventilation (days) & $1(1-3)$ & $2(1-3)$ & 0.7401 \\
Discharge (days) & $7(6-8)$ & $7(5-8)$ & 0.7687 \\
Death & 0 & $8 \%$ & 0.8423
\end{tabular}

Results are expressed as median and lower/upper quartiles. Mann-Whitney U test and Fisher's exact test were used to compare data between the two groups.

In the CPB group operations were undertaken for atrial septal defect $(n=13)$, ventricular septal defect $(n=16)$, tetralogy of Fallot $(n=6)$, simple arterial transposition $(n=11)$, atrioventricular canal $(n=6)$, total anomalous pulmonary venous connection $(n=3)$, pulmonary artery conduit $(n=$ $2)$, anomalous coronary artery $(n=1)$, atrial switch $(n=1)$, mitral valve replacement $(n=1)$, pulmonary artery debanding $(n=1)$, aortic valve replacement $(n=1)$, aneurysm of the membranous septum $(n=1)$, and atrial septectomy $(n=1)$.

Serial changes in biochemical markers. Serial changes in myoglobin, CK-MB, cTnT, and cTnI in the control and CPB groups are illustrated in Fig. 1, $A$ to $D$, respectively. So that comparative analysis could be done, the median and upper and lower quartiles for each biochemical marker at a specific time point and the total AUC are given in Table III.

Control group. In the control group there were highly significant $(p<0.01)$ increases in myoglobin (Fig. 1, $A$ ) and CK-MB (Fig. 1, $B$ ) but no increase in cTnT (Fig. 1, C) or cTnI (Fig. 1, D). Myoglobin reached peak concentrations 1 to 6 hours after the operation and decreased by 24 hours to reach preoperative values by 48 hours. In contrast, CK-MB peaked between 6 and 24 hours after the operation, remained significantly elevated at 48 hours, and returned to preoperative values by 72 hours.

$C P B$ group. The CPB group showed highly significant increases in all four biochemical markers (Fig. $1, A$ to $D$ ) but with differing temporal patterns. Similar size and temporal changes in myoglobin in the control and $\mathrm{CPB}$ groups (Fig. 1, $A$ ) resulted in similar AUCs. The magnitude of increase in CK-MB (Fig. 1, B) was significantly greater between 1 and 6 hours, but not between 24 and 72 hours, in the CPB group, resulting in a significantly greater AUC compared with that of the control group. In contrast to patients in the control group, who showed no elevation in $\mathrm{cTnT}$ or cTnI, there were highly significant increases in both cardiac troponins (Fig. 1, $C$ and $D$ ), reaching a peak at 6 hours. Subsequently, cTnI showed a more rapid fall from peak levels than cTnT, so that although both cTnT and cTnI remained significantly elevated at 72 hours, the magnitude of this increase over preoperative levels was less for cTnI (approximately six times) than for cTnT (approximately 30 times).

Variables influencing release of the biochemical markers after CPB. Variables influencing the release of the biochemical markers after $\mathrm{CPB}$ are presented in Tables IV, $A$ to IV, $D$.

Younger age was a highly significant explanatory variable for the release of $\mathrm{cTnT}$ and cTnI in both bivariate and multivariate analysis (Tables IV, $A$ to IV, $D$, Figs. 2 and 3) but not for myoglobin or CK-MB. Ischemic time was a significant explanatory variable for the release of CK-MB, cTnT, and cTnI but not for myoglobin. The presence of an atriotomy or ventriculotomy was a significant explanatory variable for the release of cTnT and cTnI but not myoglobin or CK-MB, whereas repair of a VSD was a significant explanatory variable for the release of CK-MB and cTnI. Neither CPB time nor temperature was an explanatory variable for the release of any biochemical marker.

Clinical outcome. Five patients from the CPB group (8\%) died at the end of the operation or in the early postoperative period. Three had undergone an arterial switch operation, one an atrial septectomy, and one an atrioventricular canal repair. These patients' operations were all characterized by significantly longer ischemic times than those of the patients who survived ( 90 [ 65 to 99] vs 35 [23 to 47 ] minutes). In the CPB group the median (and upper and lower quartiles) period of ventilation was 2 days ( 1 and 3 days) and the mean hospital stay was 7 days (5 and 8 days).

\section{Discussion}

Before the results of our study are discussed, it is appropriate to comment on its rationale and limitations. Measurement of cardiac performance is probably the best method of assessing myocardial injury, but it is difficult in clinical practice ${ }^{11}$ and may not distinguish between functional impairment and subtle levels of irreversible injury. Biochemical markers are easily measured from blood samples and detect even minor levels of myocardial injury in all patients. Caveats concerning the myocardial specificity of biochemical markers after cardiac surgery (e.g., 


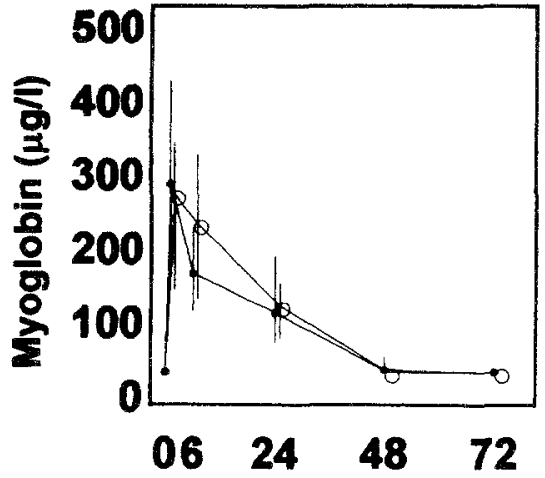

A

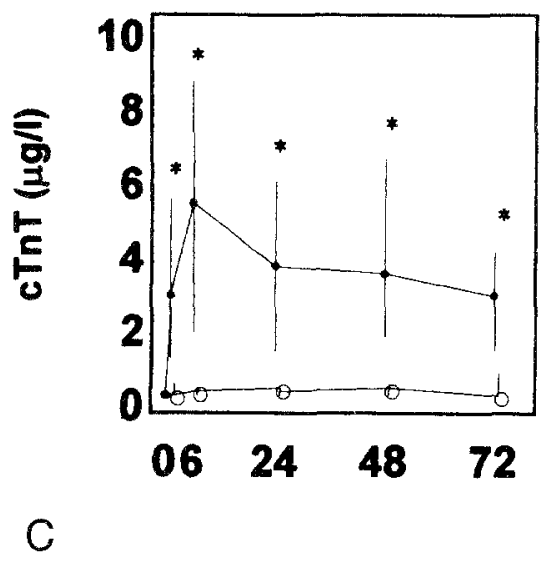

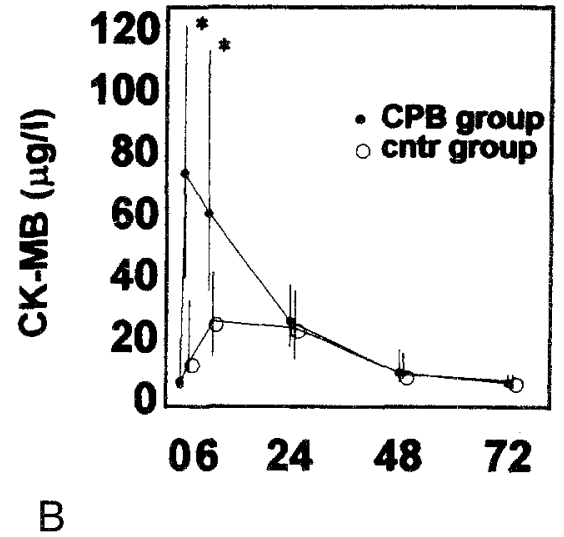

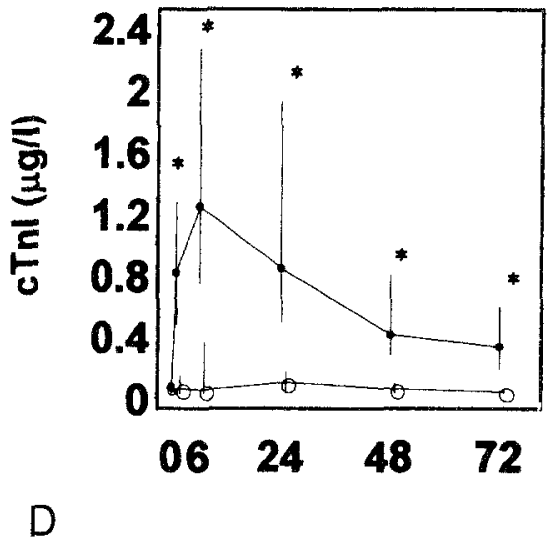

\section{time (hours)}

Fig. 1. Serial changes in myoglobin (A), CK-MB (B), cTnT (C), and cTnI (D) in the control and CPB groups. Results expressed as median and lower/upper quartiles. ${ }^{*} p<0.05$ between groups at corresponding time points.

myoglobin and CK-MB are released predominantly but not exclusively from myocardium after cardiac surgery) can be resolved by the measurement of cTnT and cTnI, highly specific myocardial isoforms of the respective subunits of the troponin regulatory complex. In a preliminary report, we $e^{9}$ confirmed the cardiac specificity of these markers after pediatric cardiac surgery and suggested that release of troponins might be greater after pediatric than adult cardiac surgery. In that study, however, there were insufficient patients to investigate the effects of age and ischemic time on troponin release.

The major limitations of this study, as with most studies of myocardial injury after pediatric cardiac surgery, are the heterogeneous nature of the patients with their differing underlying pathophysiologic processes and the various types of operations performed. Nevertheless, the current study was of sufficient size to allow some valuable conclusions to be drawn regarding myocardial specificity of the various biochemical markers, the magnitude and temporal pattern of serial changes in their plasma concentrations after cardiac surgery, and the effects of age and ischemic time on their release.

The results of our study suggest the following:

1. cTnT and cTnI are specific markers of myocardial injury after pediatric cardiac surgery. Whereas myoglobin and CK-MB increased significantly in both the control and the CPB groups, increases in cTnT and cTnI occurred only in the CPB group.

2. Although congenital heart disease may result in abnormal myocardial function, particularly in pressure- or volume-overloaded hearts (or both), the normal serum levels of myoglobin, CK-MB, cTnT, 
Table III. Time changes of biochemical markers of myocardial injury in pediatric cardiac surgery

\begin{tabular}{|c|c|c|c|c|c|c|c|}
\hline \multirow[b]{2}{*}{ Variable } & \multirow[b]{2}{*}{ Sample size } & \multicolumn{2}{|c|}{$\begin{array}{l}\text { CBP group } \\
(n=64)\end{array}$} & \multicolumn{3}{|c|}{$\begin{array}{l}\text { Control group } \\
(n=16)\end{array}$} & \multirow[b]{2}{*}{ p Value } \\
\hline & & Median & $Q 25$ & Median & $Q 25$ & $Q 75$ & \\
\hline \multicolumn{8}{|l|}{$c \operatorname{Tn} T(\mu \mathrm{g} / L)$} \\
\hline Preoperative & 80 & 0.1 & 0.1 & 0.1 & 0.1 & 0.1 & \\
\hline $1 \mathrm{hr}$ & 79 & 2.8 & 1.1 & 0.1 & 0.1 & 0.4 & \\
\hline $6 \mathrm{hr}$ & 78 & 5.3 & 1.8 & 0.2 & 0.1 & 0.4 & \\
\hline $24 \mathrm{hr}$ & 75 & 3.6 & 1.3 & 0.2 & 0.1 & 0.3 & \\
\hline $48 \mathrm{hr}$ & 75 & 3.4 & 1.7 & 0.3 & 0.1 & 0.4 & \\
\hline $72 \mathrm{hr}$ & 75 & 2.8 & 1.3 & 0.1 & 0.1 & 0.7 & \\
\hline$c T n T-A U C(\mu g \times h r / L)$ & 75 & 262 & 108 & 10 & 7 & 20 & $<0.0001$ \\
\hline \multicolumn{8}{|l|}{$\operatorname{cTnI}(\mu \mathrm{g} / \mathrm{L})$} \\
\hline Preoperative & 80 & 0.05 & 0.01 & 0.02 & 0.01 & 0.05 & \\
\hline $1 \mathrm{hr}$ & 79 & 0.79 & 0.45 & 0.04 & 0.01 & 0.12 & \\
\hline $6 \mathrm{hr}$ & 78 & 1.22 & 0.72 & 0.03 & 0.01 & 0.34 & \\
\hline $24 \mathrm{hr}$ & 75 & 0.82 & 0.47 & 0.08 & 0.01 & 0.15 & \\
\hline $48 \mathrm{hr}$ & 75 & 0.39 & 0.26 & 0.04 & 0.01 & 0.07 & \\
\hline $72 \mathrm{hr}$ & 75 & 0.31 & 0.17 & 0.02 & 0.01 & 0.03 & \\
\hline$c T n I-A U C(\mu g \times h r / L)$ & 75 & 56 & 37 & 4 & 1 & 7 & $<0.0001$ \\
\hline \multicolumn{8}{|l|}{$\mathrm{CK}-\mathrm{MB}(\mu \mathrm{g} / \mathrm{L})$} \\
\hline Preoperative & 80 & 3.5 & 2.3 & 2.7 & 2.4 & 3.6 & \\
\hline $1 \mathrm{hr}$ & 79 & 71.0 & 37.3 & 9.9 & 6.5 & 30.3 & \\
\hline $6 \mathrm{hr}$ & 78 & 58.4 & 33.0 & 23.1 & 12.3 & 39.2 & \\
\hline $24 \mathrm{hr}$ & 69 & 23.0 & 15.2 & 20.7 & 10.8 & 33.4 & \\
\hline $48 \mathrm{hr}$ & 69 & 6.5 & 3.7 & 6.1 & 4.5 & 13.1 & \\
\hline $72 \mathrm{hr}$ & 69 & 3.3 & 2.5 & 3.9 & 1.9 & 6.0 & \\
\hline$C K-M B-A U C(\mu g \times h r / L)$ & 69 & 1770 & 1162 & 1058 & 646 & 1851 & 0.0190 \\
\hline \multicolumn{8}{|l|}{ MYOGLOBIN $(\mu \mathrm{g} / \mathrm{L})$} \\
\hline Preoperative & 37 & 25 & 25 & 25 & 25 & & \\
\hline $1 \mathrm{hr}$ & 36 & 279 & 168 & 264 & 136 & 335 & \\
\hline $6 \mathrm{hr}$ & 36 & 158 & 108 & 225 & 124 & 319 & \\
\hline $24 \mathrm{hr}$ & 36 & 105 & 65 & 115 & 71 & 145 & \\
\hline $48 \mathrm{hr}$ & 36 & 28 & 25 & 25 & 25 & 25 & \\
\hline $72 \mathrm{hr}$ & 36 & 25 & 25 & 25 & 25 & 25 & \\
\hline$A U C(\mu g \times h r / L)$ & 36 & 6807 & 5176 & 7713 & 4111 & 9071 & 0.6650 \\
\hline
\end{tabular}

Mann-Whitney U test was used to compare AUC between the two groups. The $p$ values in the last column represent AUC differences between the two groups. Time-point marker values indicate the values used for the calculation of AUC. $C P B$, Cardiopulmonary bypass; $c T n T$, troponin T; $c T n I$, troponin I; $A U C$, area under curve (over 72 hours); $Q 25, Q 75$, lower, upper quartiles; $N S$, not significant.

and $\mathrm{cTnI}$ before operation suggested that there was no ongoing structural damage. On the other hand, these dilated, hypertrophied, and hypoxic hearts may be more sensitive to the effects of ischemia and reperfusion.

3. The magnitude and temporal release of the various biochemical markers differed within and between the control and CPB groups. Whereas serial changes in myoglobin were very similar in the control and CPB groups, leading to a similar AUC, a threefold increase in CK-MB in the CPB group compared with the control group between 1 and 6 hours led to a doubling of the AUC despite similar concentrations between 24 and 72 hours. CTnT and cTnI were elevated only in the CPB group, with peak concentrations being reached at 6 hours; in contrast, concentrations of myoglobin and CK-MB peaked at 1 hour. Furthermore, whereas myoglobin and CK-MB had returned to preoperative concentrations by 72 hours, both cTnT and cTnI remained significantly elevated at 72 hours, with the respective increases over preoperative concentrations being approximately sixfold for $\mathrm{cTnI}$ and thirtyfold for cTnT.

4. Multiple regression analyses showed that younger age correlated significantly with an increased release of cTnT and cTnI (but not myoglobin or CK-MB) and was particularly marked in children less than 12 months of age. Inasmuch as there is no evidence that the plasma clearance of cardiac troponins (or myoglobin or CK-MB) is different in younger patients, this implies a true 


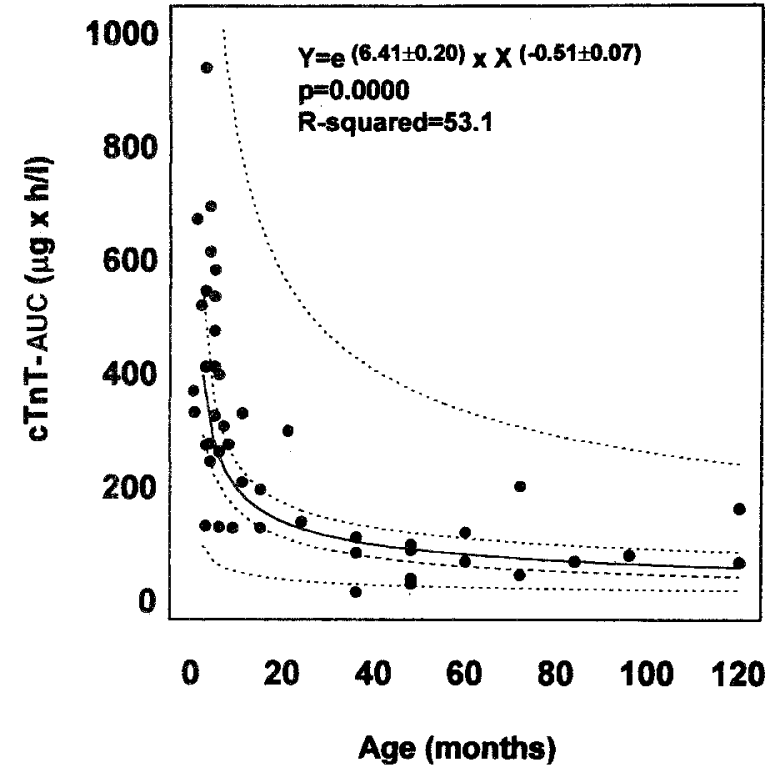

Fig. 2. Relationship between postoperative $c \operatorname{Tn} T$ release, as expressed by the AUC, and age in pediatric cardiac surgery. Data analyzed by nonlinear regression analysis and analysis of variance for the full regression provided a $p$ value $<0.001$. Dotted lines denote the $95 \%$ confidence and prediction limits.

increase in myocardial injury in these patients. Whether this represents differing underlying pathophysiologic processes in the younger age group or an increased vulnerability of immature myocardium to the ischemia-reperfusion injury of cardiac surgery is not clear from our study.

5. Duration of ischemic time, expected to be the strongest predictor of release of biochemical markers, correlated most strongly with the release of cTnT and cTnI, more weakly with the release of CK-MB, and not with that of myoglobin. This is consistent with the increased specificity of the cardiac troponins.

6. The presence of an atriotomy or ventriculotomy (or both) was itself a significant explanatory variable within the multiple regression analysis model for the release of cTnT and cTnI, presumably because of their increased cardiac specificity. We have no obvious explanation for the observation that operation for ventricular septal defect predicted release of CK-MB and $\mathrm{cTnI}$ but not cTnT.

In comparison with previous studies in which we measured concentrations of myoglobin, CK-MB, and $\mathrm{cTn} T$ (but not $\mathrm{cTnI}$ ) in adults, ${ }^{12-14}$ the temporal

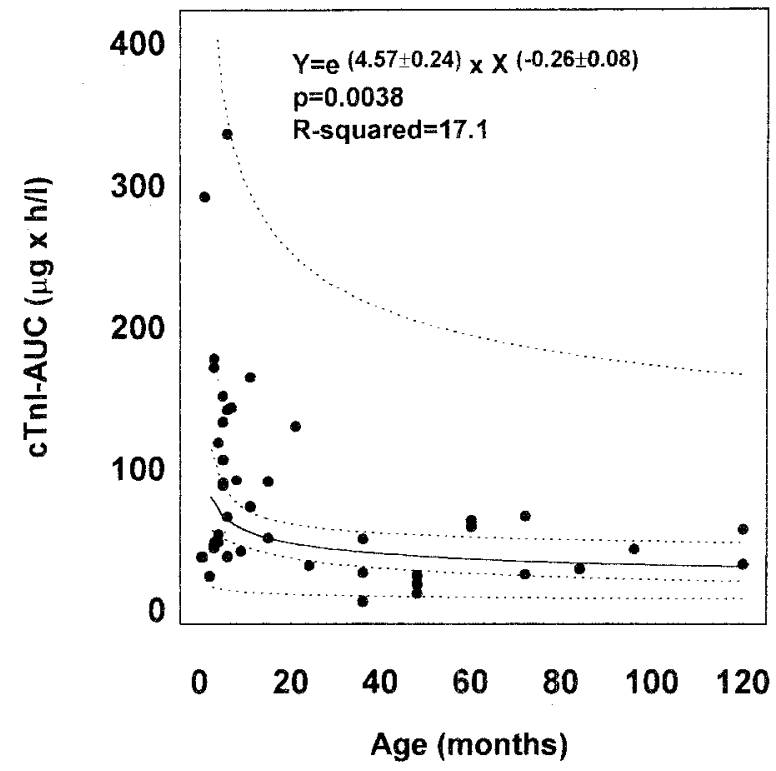

Fig. 3. Relationship between postoperative cTnI release, as expressed by the AUC, and age in pediatric cardiac surgery. Data analyzed by nonlinear regression analysis and analysis of variance for the full regression provided a $p$ value $=0.004$. Dotted lines denote the $95 \%$ confidence and prediction limits.

response of the same biochemical markers, in terms of peaks and return to preoperative concentrations, was very similar to that in our pediatric population. However, whereas the peak myoglobin concentration was similar in adults and children, the peak CK-MB and cTnT concentrations were approximately three times greater in children and significantly exceeded the limits for detecting clinically relevant myocardial injury. ${ }^{15}$ Conclusions regarding the implied differing vulnerability of adult and pediatric myocardium should be interpreted cautiously because of the differences in the underlying pathophysiologic processes, with those of adults being predominantly ischemic and those of the younger population being pressure-volume overload.

Cardiac troponins are located within myocytes both in a small free cytosolic pool and in a larger structurally bound fraction, at least in adults. ${ }^{16} \mathrm{~A}$ proportion of circulating cardiac troponins detectable in the first few hours after severe ischemia may be due to leakage from the cytosolic pool and may indicate reversible cell membrane injury. Inasmuch as the half-life of cTnT is only 2 hours, because of rapid plasma clearance, continued 
Table IV, A. Forward stepwise regression model of cTnT-AUC $(\log \mu g \times h r / L)$

\begin{tabular}{llccrc}
\hline \multicolumn{1}{c}{ Variable } & Coefficient & Standard error & $t$ & $p$ Value \\
\hline Constant & & 3.77 & 0.69 & & \\
Age & (log months) & -0.54 & 0.06 & -8.0 & $<0.0001$ \\
Ischemia time & (log minutes) & 0.61 & 0.15 & 3.9 & 0.0003 \\
A \pm V & (yes $=1$, no $=0)$ & 0.72 & 0.28 & 2.6 & 0.0139 \\
\hline
\end{tabular}

Adjusted $R^{2}=0.66$; residual standard error $=0.60$; analysis of variance for the full regression $p<0.0001$. cTnT-AUC, age, and ischemia time underwent a logarithmic transformation to achieve suitable linear relationships between the dependent variable and each continuous explanatory variable $c T n T-A U C$, Troponin $\mathrm{T}$ area under curve (over 72 hours); $A \pm V$, atriotomy \pm ventriculotomy.

Table IV, B. Forward stepwise regression model of cTnI-AUC $(\log \mu g \times h r / L)$

\begin{tabular}{llcccc}
\hline \multicolumn{1}{c}{ Variable } & & Coefficient & Standard error & $t$ & $p$ Value \\
\hline Constant & & 1.87 & 0.76 & & \\
Age & (log months) & -0.26 & 0.07 & -3.45 & 0.0013 \\
Ischemia time & (log minutes) & 0.49 & 0.17 & 2.87 & 0.0064 \\
A \pm V & (yes $=1$, no $=0)$ & 0.92 & 0.31 & 2.92 & 0.0057 \\
VSD & (yes $=1$, no $=0)$ & 0.63 & 0.21 & 2.89 & 0.0062 \\
\hline
\end{tabular}

Adjusted $R^{2}=0.48$; residual stanidard error -0.67 ; analysis of variance for the full regression $p<0.0001$. cTnI-AUC, age, and ischemia time tunderwent a logarithmic transformation to achieve suitable linear relationships between the dependent variable and each continuous explanatory variable. $c$ ThI-AUC, Troponin I area under curve (over 72 hours); $A \pm V$, atriotomy \pm ventriculotomy; $V S D$, ventricular septal defect.

Table IV, C. Forward stepwise regression model of $C K-M B-A U C(\log \mu g \times h r / L)$

\begin{tabular}{llcccc}
\hline \multicolumn{1}{c}{ Variable } & & Coefficient & Standard error & $t$ & $p$ Value \\
\hline Constant & & 6.17 & 0.52 & & \\
Age & (log months) & -0.07 & 0.06 & -1.03 & 0.3085 \\
Ischemia time & (log minutes) & 0.35 & 0.13 & 2.67 & 0.0109 \\
VSD & (yes $=1$, no $=0)$ & 0.55 & 0.18 & 3.03 & 0.0044 \\
\hline
\end{tabular}

Adjusted $R^{2}=0.30 ;$ residual standard error $=0.54$; analysis of variance for the full regression $p=0.0008 . C K-M B-A U C$, age, and ischemia time underwent a logarithmic transformation to achieve suitable linear relationships between the dependent variable and each continuous explanatory variable. $C K-M B-A U C$, CK-MB area under curve (over 72 hours); VSD, ventricular septal defect.

Table IV, D. Fonward stepwise regression model of myoglobin-AUC $(\log \mu g \times h r / L)$

\begin{tabular}{llccrc}
\hline \multicolumn{1}{c}{ Variable } & & Coefficient & Standard error & $t$ & $p$ Value \\
\hline Constant & & 8.67 & 0.89 & & \\
Age & (log months) & -0.09 & 0.08 & -1.16 & 0.2579 \\
Ischemia time & (log minutes) & 0.12 & 0.24 & 0.50 & 0.6225 \\
ASD & (yes $=1$, no $=0)$ & -0.30 & 0.33 & -0.90 & 0.3786 \\
\hline
\end{tabular}

Adjusted $R^{2}=0.10$; residual standard error $=0.54$; analysis of variance for the full regression $p=0.1663$. Myoglobin-AUC, age, and ischemia time underwent a logarithmic transformation to achieve suitable linear relationships between the dependent variable and each continuous explanatory variable. Myoglobin-AUC, Myoglobin area under curve (over 72 hours); $A S D$, atrial septal defect.

increases after 12 hours probably indicates true degradation of myofilaments. One possible explanation for the difference in magnitude and temporal responses of $\mathrm{cTnT}$ and $\mathrm{cTnI}$ is that $\mathrm{cTnI}$ is only expressed in cardiac muscle throughout ontogeny, whereas cTnT is expressed in fetal skeletal muscle and may be reexpressed in injured human skeletal muscle. ${ }^{17,18}$

In summary, this study supports the specificity of cTnT and cTnI as markers of myocardial injury after pediatric cardiac surgery and defines the importance of age and ischemic time in determining their release. Our results, in comparison with previous data in adults, raise the possibility that the pediatric heart may be more vulnerable to the effects of ischemia and reperfusion than the adult heart. Cardiac troponins are useful for providing comparative data and will aid assessment of new myocardial protective strategies or other potentially therapeutic myocardial interventions. 


\section{REFERENCES}

1. Kirklin JK, Blackstone EH, Kirklin JW, McKay R, Pacifico AD, Bargeron LM. Intracardiac surgery in infants under age 3 months: incremental risk factors for hospital mortality. Am J Cardiol 1981;48:500-6.

2. Yano Y, Braimbridge MV, Hearse DJ. Protection of the pediatric myocardium: differential susceptibility to ischemic injury of the neonatal rat heart. J Thorac Cardiovasc Surg 1987;94:887-96.

3. Bore EL, Gallagher KP, Drake DH, et al. The effect of hypothermic ischemia on recovery of left ventricular function and preload reserve in the neonatal heart. $\mathbf{J}$ Thorac Cardiovasc Surg 1988;95:814-8.

4. Grice WN, Konishi T, Apstein CS. Resistance of neonatal myocardium to injury during normothermic and hypothermic ischemic arrest and reperfusion. Circulation 1987;76(Suppl): V150-5.

5. Julia P, Kofsky ER, Buckberg GD, Young HH, Bugyi HI. Studies of myocardial protection in the immature heart. I. Enhanced tolerance of immature versus adult myocardium to global ischemia with reference to metabolic differences. J Thorac Cardiovasc Surg 1990;100:879-87.

6. Bull C, Cooper J, Stark J. Cardioplegic protection of the child's heart. J Thorac Cardiovasc Surg 1984;88:287-93.

7. Hammon JW Jr. Myocardial protection in the immature heart. Ann Thorac Surg 1995;60:839-42.

8. Smolenski RT, Swierczynski J, Narkiewicz M, Zydowo MM. Purines, lactate and phosphate release from child and adult heart during cardioplegic arrest. Clin Chim Acta 1990;192: $155-64$.

9. Taggart DP, Hadjinikolas L, Wong K, Yap J, Hooper J, Kemp M, et al. Vulnerability of pediatric myocardium to cardiac surgery. Heart 1996;76:214-7.
10. Shell WE, Sobel BE. Biochemical markers of ischemic injury. Circulation 1976;53(Suppl):I98-106.

11. SelI TL, Purut CM, Silva R, Jones RH. Recovery of myocardial function during coronary artery bypass grafting: intraoperative assessment by pressure volume loops. J Thorac Cardiovasc Surg 1991;101:681-7.

12. Taggart DP, Young V, Hooper J, Kemp M, Walesby R, Magee $P$, et al. Lack of cardioprotective efficacy of allopurinol in coronary artery surgery. Br Heart J 1994;71:177-81.

13. Taggart DP, Bhusari S, Hooper $J$, Kemp M, Magee P, Wright $\mathrm{JE}$, et al. Intermittent ischemic arrest and cardioplegia in coronary artery surgery: coming full circle? Br Heart J 1994;72:136-9.

14. Taggart DP, Jenkins M, Hooper J, Hadjinikolas L, Kemp M, Hue D, et al. Effects of short term supplementation of Coenzyme Q10 on myocardial protection during cardiac surgery. Ann Thorac Surg 1996;61:829-33.

15. Mair J, Wieser Ch, Seibt I, et al. Troponin $\mathrm{T}$ to diagnose myocardial infarction in bypass surgery. Lancet 1991;337: 434-5.

16. Katus HA, Remppis A, Scheffold T, Diederich KW, Kuebler W. Intracellular compartmentation of cardiac troponin $\mathrm{T}$ and its release kinetics in patients with reperfused and nonreperfused myocardial infarction. Am J Cardiol 1991;67:1360-7.

17. Adams JE, Abendschein DR, Jaffe AS. Biochemical markers of myocardial injury. Is $\mathrm{MB}$ creatine kinase the choice for the 1990s? Circulation 1993;88:750-63.

18. Bhavsar P, Dhoot GK, Cumming DV, Butler-Browne GS, Yacoub $\mathrm{MH}$, Barton OJ. Developmental expression of troponin I isoforms in fetal human heart. FEBS Lett 1991;292:5-8. 